Dear session organizers,

Authors thanks the reviewers for very useful suggestions and comments to improve the quality of the paper. The text was modified accordingly. A detailed response to reviewer comments follows.

\title{
Response to Reviewer 1
}

The authors need to carefully proofread their manuscript for grammatical errors. The articles " $a$ ", "an" and "the" are either missing or unnecessarily inserted throughout the paper. A few examples include:

Abstract, 1st sentence:insert "the" before 'low temperature combustion' (also, a note on capitalization-even though the abbreviation is capitalized, the full words are not)

Abstract, 3rd sentence:insert "the" between 'lowers' and 'rate of heat release' Introduction, 1st sentence:delete 'been' and insert " $a$ " after 'triggered' (see earlier note on capitalization as it also applies to 'internal combustion')

Be sure to double check for spelling errors as well.(Caption for Fig. 8 on p. 7:"startification" should be spelled "stratification", and p. 9 last paragraph "fist" should be spelled "first" and "form" should be spelled "from")

Some sentences are confusing and need to be clarified. A few examples include:

P. 2 paragraph after Table 1: "Izadi et al. [8] found that ignition sensitivity for the experiments as in Fig. 1."This could be edited many ways, but it seems like the authors meant to insert a clause describing some notable result from [8] before the phrase "as in Fig. 1."

P. 9 Second to last sentence in the "SOI -40" paragraph:"This is due to availability of larger amount of fuel in SOI -80 while second stage ignition."

Thanks for the detailed observation of Reviewer 1. We have amended the manuscript text accordingly.

Overall, this CFD paper helps to explain the experimental results observed in SAE Technical Paper 2016-01-0761. Note that I had to search for this paper number because the references section does not list the paper number for [8]. The authors need to be cautious about making broader, unproven claims that air-fuel mixture stratification can effectively control PPCI combustion phasing "under different engine operating conditions" (last sentence of abstract) when they only investigated a single, low-load operating condition.

Abstract was changed. Please check.

The authors acknowledge, in the last sentence of the second paragraph of the Introduction, that "There have note the correction from "has" to "have" been a number of experimental and numerical studies on the effect of mixture stratification on note the deletion of unnecessary article "the" PPCI combustion characteristics", so given that they are aware of other relevant papers it would be pertinent and informative to briefly review/compare/contrast (cite!) these works in the Introduction and discussion of their own results.

There was a misunderstanding in the first draft. After this sentence both experimental and numerical literature review were provided. Please check.

The short paragraph on "Specific Aims" contradicts the Introduction when the authors write "no detailed attention was given to the effect of fuel stratification."

The text was changed: "Although there has been a number of studies on experimental and numerical representation of the PPCI combustion, comparative studies on the effects of air-fuel stratification on PPCI combustion phasing characteristics are missing. Combustion phasing is a key concept which has direct control on output power and final emission levels. The aim of this paper is to show combustion phasing characteristics of a PPCI engine under low and high air-fuel mixture stratification levels and highlight their differences. This can further be used as a key element for PPCI engine control. " to clearly show the aim of this paper.

p. 3, last paragraph of left column and Fig. 2: The authors should briefly describe the stratification calculation so the papers stands alone and the reader doesn't have to go looking for another paper.

The text was modified and definition of the fuel stratification was included. 
p. 4, the citation for Ranz and Marshall's 1952 paper is missing.

p. 5, a citation for Spray A experiments is missing.

p. 5 paragraph following subtitle "Effects of Air-Fuel Stratification": The fourth sentence repeats the point of the second sentence in a slightly different wording, making the fourth sentence redundant and unnecessary.

The text was corrected as suggested.

p. 9 first sentence of the first paragraph, left column: The authors state that "it can be seen that mixture fraction in the low stratification case... spans a narrower range compared to the high stratification case..." From my inspection of Figs. 7 and 8 , the low stratification range is $0.001<Z<0.105$ and the high stratification range is $0<Z<0.11$. Obviously the range is narrower, but how significant is it? Perhaps the authors could show a pdf of the mixture fraction to better convey their argument. They also state "For all shown engine times of SOI -80, the note the correction by adding the word 'the' mixture fraction is lower than Zst $=0.0558 . "$ Inspection of Fig. 7 clearly shows this statement is false since all 8 subplots show distributions greater than Zst.

p. 9 second paragraph, left column: The authors state "At early crank angles...having more time for mixing has caused a note the correction by adding the word 'a' large amount of mixture fractions to have very similar temperature (around $700 \mathrm{~K}$ )." But in the previous paragraph the authors stated that the mixture fractions at $700 \mathrm{~K}$ temperature are in the crevice. This requires clarification.

p. 9 third paragraph, left column: The authors state "the maximum range of mixture fraction before ignition for SOI -40 is almost double the value in SOI -80..." My inspection of Figs. 7 and 8 differs with this conclusion. Are the authors specifically referring to the regions with the flame? The authors should clarify this statement.

Misunderstanding was happened in the first draft. As Reviewer 1 stated in the last comment, our purpose of mixture fraction range is the mixture fractions which participate in combustion and not the ones trapped in the crevice volume. Manuscript was accordingly modified to: Initially, it should be noted that there are range of mixture fractions (similar to a line) with temperature about $640 \mathrm{~K}$ for SOI -80 and 700K for SOI -40 in Fig.7 and Fig.8. These mixture fractions are the fuel trapped in the piston crevice volume that will not participate in the combustion and discussions below do not take these mixture fraction into account. Moreover, temperature difference of these trapped mixture fraction in the crevice volume of the cases is due to the minor differences of intake air temperature as stated in Table 1 for different injection timings. .

p. 9 Conclusions: With only one condition investigated in the current work, the authors should mention any future plans they have to continue their study of mechanisms to control combustion phasing in PPCI combustion.

p. 9 Conclusions: avoid informal colloquialisms such as "pretty" in describing a homogeneous mixture.

References are not in the SAE format.

The paper is missing a section on definitions/abbreviations.

As suggested we have modified the Conclusion section. SAE format now is followed for the references and a table of Abbreviations was added.

\section{Response to Reviewer 2}

The authors conducted an interesting study to compare the results of PPCI CFD simulations and optical engine visualizations of two injection timings. There is a huge potential of this work to discover correlations between actual optical engine mixture stratification levels coupled with additional information from simulations. Figures 7-10 provide interesting graphics, but there was very little discussion or "deep dive" into the information provided. Please try to dig a little deeper into the data and provide additional observations in the text on what was learned using the CFD simulations in addition to the optical engine testing. Thanks for the kind considerations. We provided some additional text in the result section of the paper mainly highlighting what can be understood numerically which is not obtainable by the experiments. More discussions were added on sequence of first and second stage ignition of the cases under study.

Please make sure that all references comply with the SAE required format in the author pack and resources website.

Page 2 of 16 
"Although there has been a number of studies on experimental and numerical representation of the PPCI combustion, no detailed attention was given to the effect of fuel stratification on the conceptual goal of PPCI combustion which is combustion control." - There has been work on the topics of fuel stratification and PPCI combustion control. Perhaps the authors can write that there has not yet been enough work in this area.

Reference section was modified to follow SAE format and manuscript text regarding the problem of availability of a few comparative studies on fuel stratification effects on PPCI combustion was modified. Please check.

"PRF70 is used as fuel in the experiments, considering its low-reactive characteristics which extends the ignition delay at elevated loads of PPCI combustion." Please do not start sentences with acronyms. Also, the authors should be clear when defining PRF70 as "low-reactive," to what that is with respect to. Perhaps they could write less reactive than diesel fuel.

Thanks for the suggestions. Manuscript text was modified.

In Table 1, the authors wrote that the intake temperature was 350-380 K (77-107 C) and the engine compression ratio was 12.6:1. These are unique conditions for a low load study of PPCI. Most PPCI engines use higher compression ratio and intake temperatures more typical of production engines (40-75 C). It would be helpful if the authors described why such operating conditions were decided on for this study, why a PRF70 fuel (which is neither representative of commercial diesel nor gasoline), and how these test conditions are relevant to the body of PPCI research currently ongoing. This would improve the long-term value of this work.

We used an optical engine which required lower compression ratios for protection of quartz parts. However, by increasing the intake temperature we could mimic proper thermodynamic conditions at TDC for chemical reactions as can also be achieved by higher CR and lower intake temperatures. PRF70 is also highly recommended for PPCI regarding its low-reactive characteristics which extends the ignition delay. This is explained within the paragraph before Table 1. Although PRF70 is not a 'viable future fuel', too expensive, the aim was to have a model fuel that can be simulated with a less dependency on combustion chemistry. The experiments serve as database for confrontation with simulations hence the choice of fuels.

Please follow SAE and scientific guidelines to use a space between numbers and units, "640 bar", etc.

Please be sure that the authors have received permission from SAE to reproduce figures from other SAE papers. Otherwise this is a copyright violation.

Please note that injection timings need to be referenced to CAD before or after TDC.

Manuscript text was modified as Reviewer 2 suggested.

Please state whether positive or negative "ignition sensitivity" corresponds with advancing or retarding CA50 in the paragraph after Table 1.

The method of determining "ignition sensitivity" is now extended in the manuscript and whenever this value gets negative values, it is regarded as unexpected ignition sensitivity.

Please explain, at least generally, what "Level of Stratification" is and how it was calculated for Figure 2. This is important information in the experimental setup of this paper and readers should not be required to read another previous paper. Also, what are cases $A, B$, and $C$ ? Why is it necessary to show three cases instead of one? What is the useful information?

More definition of the air-fuel stratification was added to the text. Cases $\mathrm{A}, \mathrm{B}$, and $\mathrm{C}$ are different in duration of injection and Case $\mathrm{C}$ has the largest duration which resulted in a higher range of air-fuel stratification. This is why Case $\mathrm{C}$ was simulated.

What was the SOI for the level of stratification plot against injection pressure shown in Figure 2? What is the injection pressure of the SOI sweeps in Figures 1 and 2?

By averaging the stratification level from -100 to -12.5 CAD for each injection case, stratification level as a function injection pressure is obtained. Lower injection pressure causes relatively higher combustion stratification, while higher injection pressure leads to more homogeneous combustion. Injection pressures of the Cases were represented in the manuscript and experimental section.

Please do not use citations as nouns in the sentence. Please cite references by the authors names, followed by a citation number (preferably at the end of the sentence before the period.

Manuscript was modified accordingly.

In these tests the engine was skip-fired every 10th cycle. How did the authors determine combustion chamber surface temperatures of 420, 460, and $470 \mathrm{~K}$ were appropriate for the model?

Page 3 of 16 
This is a very good question. From the experiments we knew the initial pressure and the mass of air trapped within the combustion chamber (with known volumetric efficiency of the engine under study). Initial air temperature had close values to the intake manifold temperature and trial and error method was used to match the trapped mass in the simulation with the experiments by minor changes on initial gas temperature of the chamber. Then the same method was applied to the cylinder wall temperatures to match the average pressure curve of the compression (without injection and combustion) from the experiments.

"This is not a strong assumption and experimentally also it was observed a few engine cranks were needed..." - Please clarify what a few engine "cranks" are.

Manuscript text was modified. Please check.

"MZWM model is based..." - Please define all acronyms with parentheses at their first definition.

It is not clear what " $Z$ " is in figures 7 and 8, nor Zst. Please explain in the paper the significance of these plots and the connection to the mixture stratification.

List of Abbreviations was added to the end of paper. Although $\mathrm{Z}$ is a conserved scalar, its higher magnitudes represents the air-fuel mixture strength. In the stratified mixtures availability of Zst, due to its highest reactivity, can alter the ignition and combustion phasing. This is why mixture fraction and temperature diagrams were presented and discussed.

"first stage ignition gets closer to the main stage ignition and the delay time in between is reduced. This practically provides a better control on the combustion for PPCI engine." The authors state that increased stratification and reduced duration between low temperature and high temperature heat release improves combustion control in PPCI. However, the authors do not discuss at what cost. There should be a discussion in the paper about whether the shorter duration between LTHR and HTHR caused increased combustion noise, heat transfer, or emissions (especially of particulate matter or NOx).

Reviewer 2 is absolutely true, however, it should be noted that making such comparisons, combustion noise and emission levels, are true when identical mass of premixed fuel for low and high stratification cases undergo the first and second stage ignition. This was impractical in our experimental setup. It was also mentioned in the paper that due to applying inevitable glassy piston for optical access, large volume was formed for piston crevice and there was always a noticeable amount of fuel trapped in this volume. This was suggested in the paper that enhanced comparative studies can be made if large crevice volumes would be avoided in PPCI engine studies.

Please add a section for definitions and symbols.

A table of abbreviations and definitions was added to the manuscript.

The AHRR of the SOI at -40 and -80 dATDC seem like they have similar CA50. If higher stratification was achieved with SOI -40 dATDC, shouldn't its combustion phasing be advanced? This is an issue that was not discussed in the paper. If combustion phasing was matched, then how? By changing intake air temperature? Was that taken into account (if needed to be) in the combustion chamber initial surface temperatures? What else was different that needs to be mentioned between the SOI -80 and -40 dATDC tests? Air mass flow rate? Equivalence ratio?

Reviewer 2 is correct, in the experiments intake air temperature is slightly changed for different injection timings in a way to have main stage of the heat release in similar engine crank angles. However, it was shown by numerical results that the speed combustion phases from SOI -40 from first to second stage ignition is faster that SOI -80. More discussions were added in this regard. Please check.

The introduction discussed the effects of injection pressure, but there were no comparisons made in the results section regarding simulation and optical comparisons for those effects.

This is correct. However, please consider that the only goal of introducing cases with different injection pressures was to show that fuel stratification increases under lower injection pressures. The aim of the paper was on the effect of fuel stratification and then attention was given to two low and high stratification levels of low injection pressure. For sure, effects of injection pressure can be matter of future studies.

In the conclusions, "MZWM model is used as PPCI combustion occurs in pretty homogeneous mixtures." - Do the authors mean in heterogeneous mixtures?

State of air-fuel mixture was the main idea of this sentence and by homogeneous we meant mixture had enough time to mix and reduce its non-homogeneity.

There were many grammar mistakes throughout the paper. Please revise and pay special attention to the use of articles (the, a, an, etc.).

Page 4 of 16 
Manuscript was extensively modified. Please check.

Based on the title, "...Extreme Charge Stratification Levels," the reviewer expected to see conditions that had commensurate levels of stratification. However, fuel injection at -40 dATDC at a low load does not seemingly qualify as an "Extreme" level of charge stratification. Please reconsider this title to more accurately describe the mixture conditions examined.

Title and the manuscript text was modified and "low and high" was used instead of "extreme".

\section{Response to Reviewer 3}

This paper presents some simulation work performed to understand how stratification (induced by changes in injection timing) impacts combustion. The paper is moderately well written, but contains a lot of conjecture. The conclusions could be much better supported through more careful analysis and explanation. The reviewer suggests the following to improve the paper:

The authors state that a flat piston bowl is used because a piston bowl rim affects ignition behavior. How is this known? Why do the authors wish to study ignition behavior with a piston that is not representative of a real engine geometry, particularly for a numerical study?

Thanks for Reviewer 3 for this observation. The main goal of using glassy flat piston was to gain easy optical access to the combustion chamber. First statement in the manuscript was not correct and was modified.

Please define ignition sensitivity more clearly. Is it computed with finite differences, some estimate of the derivatives, or in another way altogether?

To assess the ignition sensitivity, SOI has been advanced and retarded for 2 CAD for each measurement point $(2 \times 19$ extra measurement points) and CA50 variation has been measured for this small SOI differential. And then derivatives were taken based on engine time. Manuscript text was modified.

The authors need to provide more detail about the experimental setup and how it compares to the one referred to in reference [8]. The reviewer has no access to this reference, so it is unknown if the engine configurations are the same or not.

More text was added from the experimental paper, however, repetition was avoided.

Likewise, a brief explanation should be included about how Stratification Level is computed and what its physical interpretation is. Reference 8 is not yet unavailable, so it is difficult to understand what is being shown in Figure 2.

More explanation and representation of the need for stratification study was provided. Please check.

For the simulated cases, which injection pressures and quantities were chosen? Is the load the same for both cases?

It has been said in the text that Case $\mathrm{C}$ with lowest injection pressure was selected as it creates larger range of air-fuel mixture stratification. Yes, the load is identical for both low and high stratification cases.

The authors state that a shorter delay between first- and second-stage ignition improves combustion control. What is meant by combustion control? Control authority over combustion phasing? Control of cyclical variability? Control of the extent of overmixing? Control of pollutant emissions? How exactly does control depend on the delay between first-and second-stage ignition? Is there a reference that can be cited to give the interested reader more insight?

The main idea on combustion control is the main (high temperature) heat release timing which releases large amount of fuel chemical energy. In many hydrocarbon fuels the heat release pattern is in two low and high temperature stages and under IC engine conditions due to variable ambient pressure and temperature, gaining control on the sequence of second stage heat release is a key parameter to enhance engine IMEP and subsequently pollutant emission levels. Larger times between first and second stage heat release can highly alter the heat release history and subsequent emission levels and it can be subjected to the engine cycle to cycle variations. Manuscript text was modified to clearly state what was mentioned here.

The authors show what is presumably measured AHRR, but not simulated heat release trends. The conclusions would be strengthened if the reader knew that trends in experimentally determined heat release behavior are well predicted by the simulation.

We kindly believe it was other way around. Fig. 6 is simulated AHRR results. As AHRR was driven based on first law using the pressure curve, its comparison with experiments was not included to make the Fig. 6 readable in the next discussions. Pressure curves from simulations were separately compared with the experiments in Fig. 4.

How do the authors define mixture fraction $\mathrm{Z}$ in the unburned and burned gas?

Mixture fraction is a conserved scalar and is independent of combustion and is the measure of the state of air-fuel premixing, where $\mathrm{Z}=0$ is the pure oxidizer and $\mathrm{Z}=1$ is the pure fuel. In mixture fraction transport in CFD domain there is no source term due 
to combustion but there is a source term due to the spray. This source term adds in gaseous fuel mass fraction which is the value from evaporation sub-model after coupling of Lagrangian liquid droplet and Eulerian gas flow calculations.

In figures 7, 8, and 9, is it possible to identify and/or remove the parcels that are trapped in the crevice that don't participate in reactions? For example, Figure 10 suggests that more mixture is trapped in the crevice for the early injection than for the late injection. Is this the only source of fuel that does not reach first stage ignition?

First draft of the paper did not clearly discussed the fuel trapped in the crevice volume. Now this is clearly explained before the discussion of the flame structure Z-T diagrams. Overall, the fuel which is trapped in the crevice volume does not react, neither first nor second stage ignition. Reason is because it was surrounded by cold walls (constant $420 \mathrm{~K}, 460 \mathrm{~K}$, and $470 \mathrm{~K}$ ) and large amount of heat rejection from the crevice volume prevents any exothermic reactions.

In the discussion, the authors state that with the later injection timing, the presence of richer mixtures can contribute to higher reaction rates. However, the information shown in Figure 8 suggests that much of the rich mixture does not ignite. These mixtures are not visible in the crevice in Figure 10, but should otherwise be expected to undergo both first and second stage ignition. Can the authors comment on the location of these rich mixtures, and why they do not ignite?

Thanks for this detailed comment. We have changed (re-scaled to the maximum of Z in SOI -40) the mixture fraction contours and it can be seen that there is a local rich region (not in the crevice which will not participate in combustion) at the corner of the chamber above the crevice in SOI -40, whereas in SOI -80 more homogeneous mixture fractions with lower magnitudes are available. This local richer mixture fractions in SOI -40 is the location where the ignition starts as it can also be seen from the temperature contours. As we stated in the respond to the previous comment, cold walls of crevice region prevents combustion to take place in that region.

The temperatures of the non-reactive mixture appear different for the SOI -80 and the SOI -40 cases. For example, for the SOI -80 case, minimum TDC temperatures are approximately 600K, whereas for the SOI -40 case, minimum TDC temperatures are $640 \mathrm{~K}$. The authors claim that evaporative cooling impacts the SOI -40 case more than the SOI -80 case, but it is unclear how this conclusion is reached. Please clarify.

Temperature difference of these trapped mixture fraction in the crevice volume of the cases is due to the minor differences of intake air temperature as stated in Table 1 for different injection timings. Our first justification now is modified to represent that minor temperature differences in the intake charge was the reason to the non-reactive mixture fractions in the crevice to have different temperatures.

$\mathrm{CH}_{2} \mathrm{O}$ radicals form as a result of low-temperature reactions, and are consumed if/when the mixture reaches the temperature required for second-stage ignition. How is it known that the presence of $\mathrm{CH}_{2} \mathrm{O}$ acts to shorten the second-stage ignition delay, and is not formed in mixture that does not reach second-stage ignition?

$\mathrm{CH}_{2} \mathrm{O}$ is a key element of chemical kinetics mechanism pathways of hydrocarbons with double stage ignition behavior and in a large number of studies it has proved to show the transition from first to second stage ignition. In the shock tube experiments for development and validation of the chemical kinetics mechanisms, quantification of the key chemical species including the $\mathrm{CH}_{2} \mathrm{O}$ was performed under variety of equivalence ratios (or mixture fractions) and it was proved that mixtures close to stoichiometry are more reactive with higher flame speed [1,2]. The goal of representing $\mathrm{CH}_{2} \mathrm{O}$ mass fractions was to show there is higher magnitudes of this key element for relatively more reactive richer air-fuel mixtures.

The authors state that the reason for larger $\mathrm{CH}_{2} \mathrm{O}$ mass fractions observed for the early injection case is because more fuel is available for second-stage ignition. This explanation is not clear. Are the authors stating that with the early injection case, a higher proportion of the fuel undergoes first-stage ignition, therefore more fuel may be available for second-stage ignition? Or are the authors stating that more fuel is injected for the early injection case, and is therefore available? Please elaborate on this discussion.

Former statement of the Reviewer 3 is what we meant in the manuscript. For the SOI -80 as it can also be seen in the Fig. 10 a higher proportion of fuel undergoes the first-stage ignition which also strengthens the second stage. Manuscript text was modified for clearer explanation.

\section{References}

1- Friedrichs, G., Davidson, D.F. and Hanson, R.K., 2004. Validation of a thermal decomposition mechanism of formaldehyde by detection of $\mathrm{CH} 2 \mathrm{O}$ and HCO behind shock waves. Int. J. of chemical kinetics, 36(3), pp.157-169.

2- Li, J., Zhao, Z., Kazakov, A., Chaos, M., Dryer, F.L. and Scire, J.J., 2007. A comprehensive kinetic mechanism for CO, CH2O, and $\mathrm{CH} 3 \mathrm{OH}$ combustion. Int. J. of chemical kinetics, 39(3), pp.109-136. 


\title{
Numerical Investigation of PPCI Combustion at Low and High Charge Stratification Levels
}

\author{
Author, co-author list (Do NOT enter this information. It will be pulled from participant tab in \\ MyTechZone) \\ Affiliation (Do NOT enter this information. It will be pulled from participant tab in MyTechZone)
}

Copyright $\odot 2017$ SAE International

\begin{abstract}
Partially premixed compression ignition combustion is one of the low temperature combustion techniques which is being actively investigated. This approach provides a significant reduction of both soot and NOx emissions. Comparing to the homogeneous charge compression ignition mode, PPCI combustion provides better control on ignition timing and noise reduction through airfuel mixture stratification which lowers heat release rate compared to other advanced combustion modes. In this work, CFD simulations were conducted for low and high air-fuel mixture stratification cases on a light-duty optical engine operating in PPCI mode. Such conditions were experimentally achieved by injection timing and spray targeting at similar thermodynamic conditions. After validating the computed results of cylinder pressure, and $O H^{*}$ spatial distributions, differences of engine thermal load and mixture fraction distributions at first stage and second stage ignition of PRF70 fuel for these cases were compared. With the assumption of similar second stage ignition timing which is provided by intake air heating, experimental and simulation results reveal that the time between first and second stage ignition shortens and combustion phases to the main stage ignition faster in the high stratification case. Using flame structure diagrams, this was attributed to availability of larger range of mixture fractions with higher reactivity. Creating optimum air-fuel stratification then can be considered as a useful and additional controlling parameter of PPCI engine combustion phasing and subsequent emission formation.
\end{abstract}

\section{Introduction}

Need for a novel mode of combustion to meet the legislated, ultra low, future emission standards for passenger vehicles, while maintaining high fuel economy specifications, has triggered a wide range of research in the field of internal combustion engines. HCCI combustion in Diesel engines as an advanced concept, provides thermal efficiencies similar to the conventional modes of combustion, but with dramatically lower levels of NOx emissions due to lowered peak temperature [1]. Depending on possibility of achieving considerably premixed air-fuel charge, almost all types of fuel (gasoline, Diesel, and etc) can be used in HCCI mode.
Nevertheless, practical implementation of the HCCI strategy in a Diesel engine meets the same restriction found in a gasoline engine, where compression ratio and load are limited by uncontrolled auto-ignition of the fuel [2] which in extreme operating conditions can lead to heavy engine knock [3]. In case of the HCCI combustion, this is more limiting as onset of combustion and heat release is mainly controlled by fuel chemistry that is prone to cycle to cycle variations. Such shortcomings of the HCCI combustion has shifted attention to the PPCI engines. PPCI strategy, similar to HCCI, provides the requirements of the LTC but with enhanced controllability on mixture preparation and start of combustion. This was mainly achieved by significant advances in electronically-controlled, high pressure, multiple injection fuel systems [4]. Mimicking the direct-injection feature of most conventional Diesel engines, mixture preparation for PPCI combustion usually occurs through direct injection of fuel into the combustion chamber [5, 6]. Depending on fuel type, injection timing, engine thermal load, and geometrical constraint of the piston bowl, different levels of air-fuel stratification can be obtained. There have been number of experimental and numerical studies on the effect of mixture stratification on the PPCI combustion characteristics which will be briefly discussed next. Experimentally, PPCI combustion was studied by a number of researchers firstly indicating that HCCI combustion might not meet the industrial market requirements due to lack of controllability and very intense heat release. The main idea behind the concept of the PPCI engines then is to overcome HCCI engine limitation and providing wider range of its applicability by generating mixture stratification. Injection timing and strategy are the main factors to control the mixture stratification and consequently ignition timing and emissions in PPCI engine. Mixture stratification is highly dependent on the SOI due to different mixing time [7, 8]. Li et al. [7] showed that ignition timing of PPCI engine is highly sensitive to SOI whether EGR is used or not. Multiple injection strategies can also improve the mixture preparation $[9,10]$. Dempsey et al. [9] studied effects of premixed fuel preparation by implementing a double injection strategy in a light duty engine. They investigated effects of pilot injection timing, as a determining factor of mixture homogeneity, on engine performance and emissions.

Using multi-dimensional CFD, PPCI combustion has been investigated by a number of researchers. Tamagna et al. [11] inves- 
tigated PPCI and dual-fuel combustion for different fuel reactivity levels. The Shell-CTC model in conjunction with KIVACHEMKIN model (well-mixed chemistry assumption) was used in order to account for combustion for a PPCI engine. It was concluded that including a chemical kinetics mechanism was necessary for accurate predictions of ignition delay and heat release history of PPCI combustion. Kokjohn et al. [12] have studied dualfuel PPCI engine using well-mixed chemistry formulation. In their experimental configuration, $n$-heptane was injected, as pilot fuel, to ignite premixed iso-octane. It has been concluded that for all the injection timings and operating cases ignition was started from the highest local concentration of the n-heptane. Pal et al. [13] investigated PPCI combustion using extended RIF model including spray evaporation effects in reaction space in conjunction with multi-zone well-mixed model. It was concluded that applied models have almost similar results under low air-fuel stratification condition, however, flamelet based model had more accurate results under high fuel stratification levels. Kong et al. [14] studied PPCI engine combustion using RIF model and a well-mixed model. The KIVA-RIF model predicted slightly higher in-cylinder gas temperatures that resulted in higher $N O_{x}$ emissions. Both models also predicted the benefits of late injection to soot emissions as a result of LTC concept.

\section{Specific Aims}

Although there has been a number of studies on experimental and numerical representation of the PPCI combustion, comparative studies on the effects of air-fuel stratification on PPCI combustion phasing characteristics are missing. Combustion phasing is a key concept which has direct control on output power and final emission levels. The aim of this paper is to show combustion phasing characteristics of a PPCI engine under low and high air-fuel mixture stratification levels and highlight their differences. This can further be used as a key element for PPCI engine control.

\section{Engine Setup and Operating Condi- tions}

PPCI engine experiments were conducted in Eindhoven University of Technology on a light-duty Volvo D5 Diesel engine [8]. The engine was modified for optical access from cylinder side and bottom for investigating effects of the SOI on ignition sensitivity of PPCI. As images were taken from the bottom of the cylinder a flat glassy piston was used to provide optical access to the combustion chamber. Applied fuel for the experiments was PRF70 which is less reactive than conventional Diesel fuel. This less-reactive characteristic of the applied fuel extends the ignition delay at elevated loads of PPCI combustion. Engine specifications and operating conditions were shown in Table 1 . The engine was motored at $1200 \mathrm{rpm}$ by using a dynamometer. An artificial EGR system was used to dilute the intake air by adding nitrogen. Intake pressure and temperature were also controlled using a compressor and heater. Intake air temperature was slightly changed for different injection timings to have similar engine time of the second stage (main stage) ignition.

To investigate effects of injection timing, SOI was swept from
Table 1: Engine specifications operating under PPCI mode[8]

\begin{tabular}{lc}
\hline Bore $\times$ Stroke & $81 \times 93.2 \mathrm{~mm}$ \\
Connecting rod length & $147 \mathrm{~mm}$ \\
Compression ratio & $12.6: 1$ \\
Swirl ratio & 1.8 \\
Speed & $1200 \mathrm{rpm}$ \\
IMEP (gross) & $4.3 \pm 0.9 \mathrm{bar}$ \\
Number of injector holes & 7 \\
Spray pattern included angle & $140^{\circ}$ \\
Intake pressure & $1.2 \mathrm{bar}$ \\
Intake temperature & $350-380 \mathrm{~K}$ \\
Intake Oxygen percentage & $17.6 \%$ \\
Injected fuel mass & $16 \mathrm{mg} / \mathrm{cycle}$ \\
\hline
\end{tabular}

-100 to -20 CAD ATDC for three different single-injection pressures of 640 bar (Case A), 500bar (Case B), and 420bar (Case C) with injection duration of $5 \mathrm{CAD}, 6 \mathrm{CAD}$, and $7 \mathrm{CAD}$, respectively. Experimentally, the engine was operated in a skip-fire mode (once every $10^{\text {th }}$ engine cycle) to reduce the required cleaning frequency of the optical piston and liner, and the risk of liner failure due to thermal and mechanical stresses. To assess the ignition sensitivity, SOI has been advanced and retarded for 2 CAD for each measurement point $(2 \times 19$ extra measurement points $)$ and CA50 variation has been measured for this small SOI differential. Izadi et al. [8] then defined the ignition sensitivity as the ratio of CA50 (Crank Angle 50) differential to SOI differential and quantified it for the different injection timing experiments as shown in Fig. 1. At intermediate SOI from -60 to $-25 \mathrm{CAD}$, ignition sensitivity acts unexpectedly (negative values), particularly around -50 and -30 CAD where the combustion was advanced by retarding the injection timing [8]. Considering a hypothesis of "later injection, later combustion" for late injection timing, two unexpected regions were highlighted in Fig. 1.

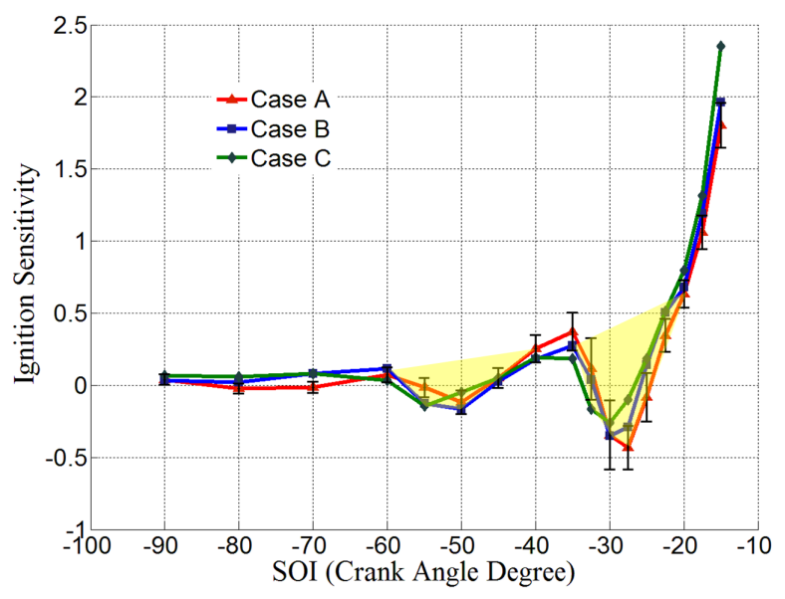

Figure 1: Ignition sensitivity as a function of injection timing for different injection strategies of PPCI combustion for Case A, B, and $\mathrm{C}$ reported by Izadi et al. [8]

Izadi et al. discussed that early injection timing (-100 CAD) causes liquid fuel to hit the liner and by the end of injection, this liquid stain on the liner evaporates after some crank angle degrees. Due to experimental limitations, the glass piston had a larger crevice volume compared to the metal piston and this 
caused trapping of a considerable amount of evaporated fuel in the piston crevice which cannot participate in combustion. Sprayliner impinging reduces with further retarding the SOI due to elevated cylinder pressure at late engine crank angles. For injection timing from -60 to -45 CAD ATDC, there was the maximum amount of trapped fuel in the crevice volume due to piston position and injection angle, causing poor combustion efficiency and low ignition sensitivity. For injection later than -50 CAD ATDC, the liquid spray did not hit the liner. However, for injection from 35 to $-25 \mathrm{CAD}$, liquid spray hit the piston crown in relatively cold thermal situations, causing poor mixing and low ignition sensitivity. These unexpected regions were shown in yellow areas in the Fig. 1.

Combustion stratification levels of the experimental cases for different start of injection times were shown in Fig.2. The methodology to calculate these stratification levels was based on angular stratification driven from $O H^{*}$ chemiluminescence images analyzed in a cylindrical coordinate system [8]. The intensity was radially averaged and plotted as a function of polar angle. Based on the minimum and maximum intensities combustion stratification was quantified for each operating conditions.

Experimental data shows that stratification of the Case $C$ was the highest among two other cases and this case was used to perform numerical simulations because its larger range of air-fuel stratification. In the numerical simulations no crevice flow model and blow-by were considered, although large crevice volume can possibly create blow-by and loss of mass. To minimize mentioned issues numerical simulations were conducted for start of injection at -80 and -40 CAD ATDC. Based on represented experimental data in Fig. 1, these injection timings were not in the highlighted zone with unexpected ignition sensitivity behavior. Hence, numerical study can mainly focus on the level of stratification of the cases.

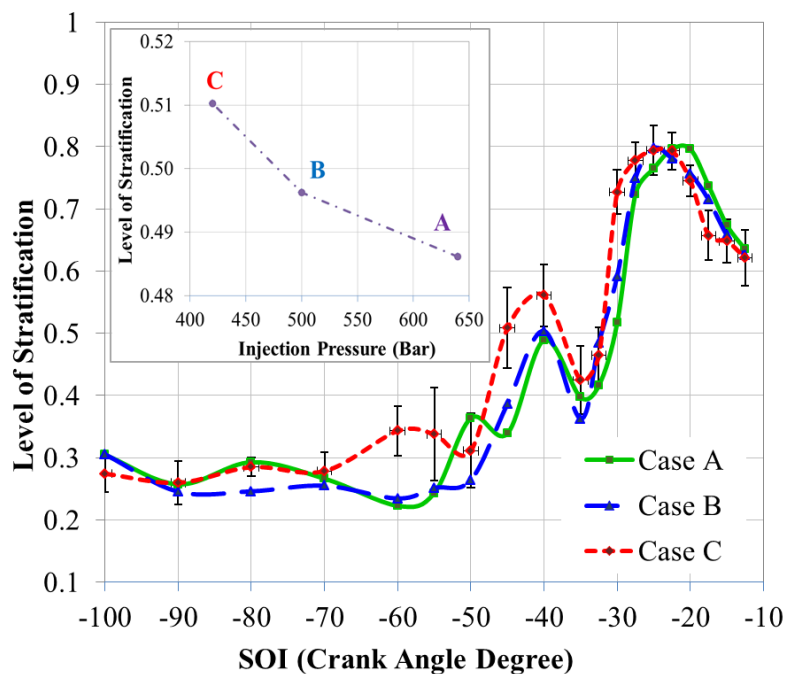

Figure 2: Stratification level of PPCI combustion of Case A, B, and $\mathrm{C}$ as a function of injection timing reported in [8]

\section{Modeling Methods}

Multi-dimensional CFD simulations for the considered cases of SOI -80 ATDC and SOI -40 ATDC as low and high air-fuel strati- fication cases were performed. Grid generation, numerical framework, applied combustion model will be represented in this section.

\section{Automatic Mesh Generation}

Mesh generation was carried out for the engine with the specification in Table 1 and it can be seen in Fig. 3 at TDC. A Spray Oriented sector grid was generated with considering the crevice volume (to match the engine compression ratio) and seven injector nozzles. Mesh generation was performed based on automatic grid generation tool [15]. This method of grid generation keeps the grid fixed on top of the combustion chamber and aligned to the spray injection axis. Layers of the cells then can be added/removed below these fixed grids during the piston movements. For more detailed explanations of the grid generation tool see [15]. For the PPCI combustion with very early injection timings, spray oriented grid will be very beneficial to have more accurate air-fuel mixing predictions. Periodic boundary condition was considered on side faces for the mesh. Cylinder head, piston, and liner wall temperatures were set to $420 \mathrm{~K}, 460 \mathrm{~K}$, and $470 \mathrm{~K}$, respectively. These temperature values were obtained by matching the trapped air mass in the simulations with air flow rate of the experiments and performing motoring simulations to accurately predict experimental motoring pressure curves.

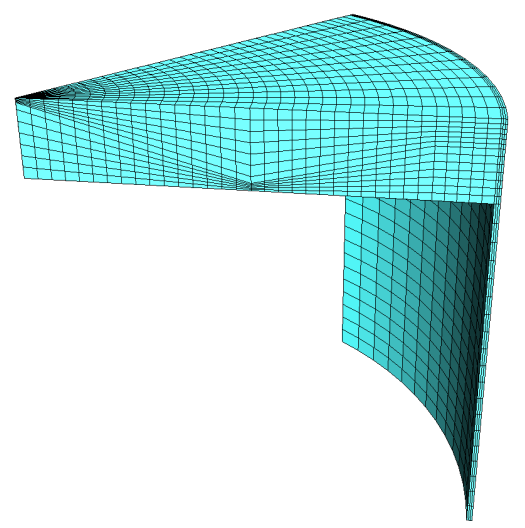

Figure 3: Spray oriented sector grid for the PPCI engine combustion simulations at TDC

\section{Numerical Framework}

Numerical simulations were conducted using the OpenFOAM ${ }^{\circledR}$ open-source code [16] in conjunction with Lib-ICE which is a set of libraries and solvers for comprehensive IC engine simulations developed by Internal Combustion Engine Group of Politecnico di Milano [17]. Turbulence was accounted by Reynolds Averaged Navier Stokes (RANS) formulation and was modeled using standard $\mathrm{k}-\varepsilon$ model. Wall boundary layer treatment for heat transfer calculations was modeled by the Huh-Chang wall function [18]. Since fuel was directly injected in the PPCI engine experiments, Lagrangian-Eulerian approach was used to model the spray evolution. The spray parcels evolve into the computational domain according to the mass, momentum and energy exchange with the continuous gas phase which was treated in an Eulerian way. In 
this work, as the PRF70 was applied in the experiments, the liquid spray was considered to contain n-heptane and iso-octane components. Spray sub-models employed in this work were the same as used by Maghbouli et al. [19]: jet and droplet breakup were computed by the KHRT model, which accounts for both KelvinHelmholtz (KH) and Rayleigh-Taylor (RT) instabilities [20]. No wall film model was applied in the simulations and impinged Lagrangian parcels considered to either bounce back to the chamber or at lower droplet mass thresholds, were removed from the wall and their mass were added to the Eulerian gaseous fuel. This was not a strong assumption and experimentally also it was observed a few engine cranks ( 1 to 4 ) were needed for impinged fuel particles to evaporate. Droplet evaporation was computed on the basis of the $D^{2}$ law and the Spalding mass number [21] while the Ranz-Marshall correlation [21] was used to model heat transfer between liquid and gas phases. Collision was neglected since it plays a minor role in evaporating sprays.

\section{Combustion Model}

The Multi-Zone Well-Mixed model was used as the combustion model and Liu's PRF mechanism [22] with 41 chemical species and 124 chemical reactions was used as reaction mechanism. MZWM model is based on well-mixed combustion model which treats each CFD cell as a closed homogeneous reactor and computes the chemical species reaction rates accordingly. Temperature, pressure and the chemical composition of the each cell were passed to a stiff ODE solver to integrate and calculate reaction rates during the given time-step to update combustion source terms in species mass and energy conservation equations. Although sub-grid turbulence-chemistry interaction is not considered in the MZWM model, when applied to the PPCI combustion it was expected to provide a reasonable estimation of ignition time and burning rate. In other words, comparing to the conventional Diesel combustion, as generally PPCI ignition occurs much later than end of injection, scalar dissipation rate in the air-fuel mixture drops to very low values and gets very close to well-mixed assumption. This is why many researchers reported that results of the models with embedded sub-grid scale turbulent mixing do not differ a lot when compared to the well-mixed combustion models $[14,13]$.

For the applied well-mixed model in this study computational efficiency was achieved by utilizing the multi-zone chemistry approach originally presented by Aceves et al. [23]. According to user-specified tolerances, zones were created by clustering CFD cells with similar temperature and equivalence ratio. Chemistry was then integrated only once per zone and reaction rates were mapped back to the CFD domain by employing a technique which preserves local cell composition and chemical elements. For more descriptions of MZWM model see [24].

\section{Results}

\section{Model Validation Study}

Initially, in-cylinder pressure results of SOI -80 and -40 ATDC were compared with experimental data in Fig. 4. These two cases represent low and high mixture stratification levels as depicted in the experimental results of Fig.2. Figure 4 also shows that MZWM model is capable of acceptable predictions for the PPCI combustion with low and high level of air-fuel mixture stratification. It then would be worth studying the mechanism of combustion initiation and progress with noticeably different stratification levels.
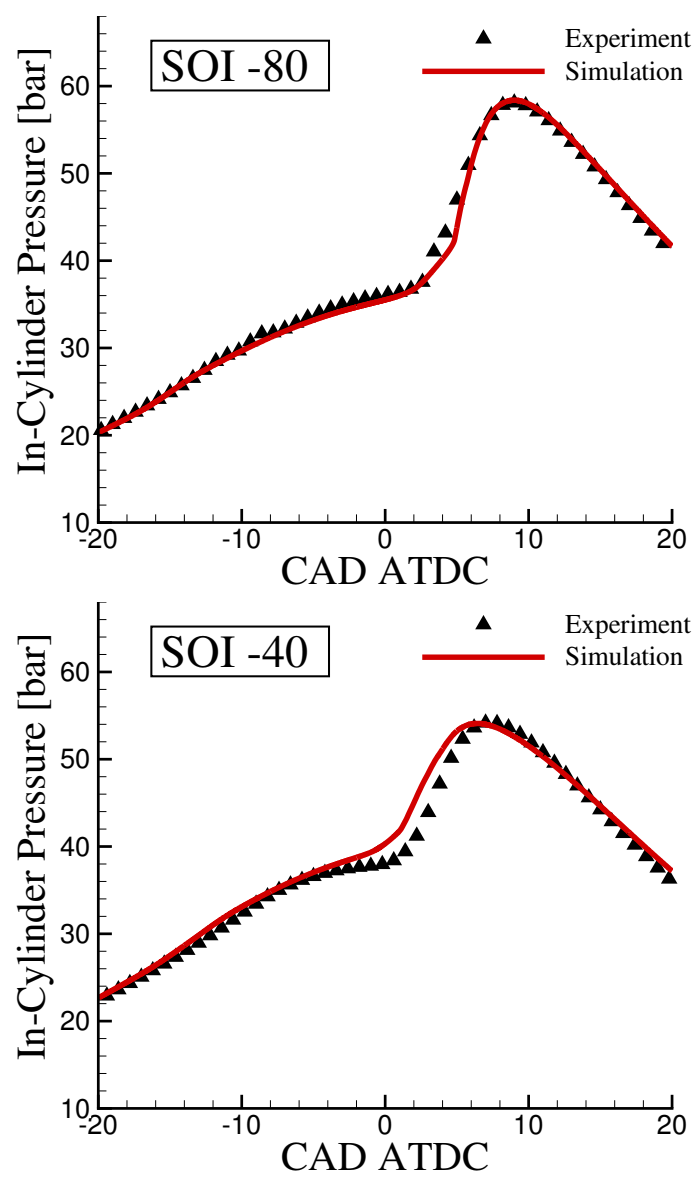

Figure 4: In-cylinder pressure comprason with experimental data for SOI of -80 and -40 ATDC.

\section{$O H^{*}$ Chemiluminescence as the Marker of the First Stage Ignition}

In the experiments, chemiluminescence was captured which corresponds to $\mathrm{OH}^{*}$ as good indicator of the ignition process under PPCI engine condition. $\mathrm{OH}^{*}$ chemistry pathways were added to the applied chemical kinetics mechanism enabling introduction of $\mathrm{OH}^{*}$ mass fraction as a transportable scalar. Figure 5 represents images (top view) of the D5 engine combustion chamber at the time of the first stage ignition: -16 CAD ATDC for SOI -80 and -12 CAD ATDC for SOI -40. Magnitudes of $\mathrm{OH}^{*}$ were normalized to the maximum value in the experiments in each image and in the CFD simulations. MZWM model was able to predict spatial distribution of $\mathrm{OH}^{*}$ and its local peak compared to the experiments. 

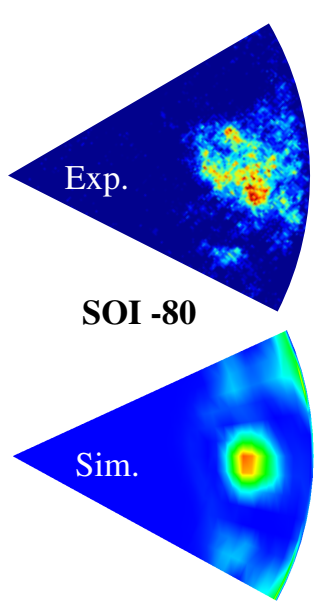

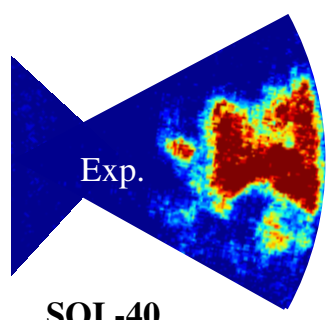

SOI -40

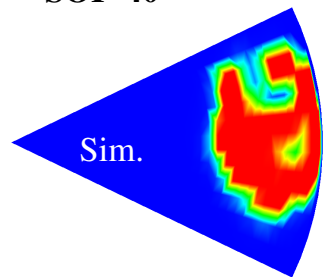

Figure 5: Experimental and numerical $O H^{*}$ distributions (scalaed to the maximum experimental values for each image) at the sequence of the first stage ignition.

\section{Effects of Air-Fuel Stratification}

Numerical results of AHRR was compared for the considered cases in Fig. 6. It can be noted that the time delay between first and second stage ignition was getting shorter by further retarding the start of injection from -80 to -40 CAD ATDC. In other words, it shows that combustion phases faster to the second and main stage heat release in higher air-fuel stratification case. The reason for this behavior will be discussed in flame structure, mixture fraction-temerature, diagrams.

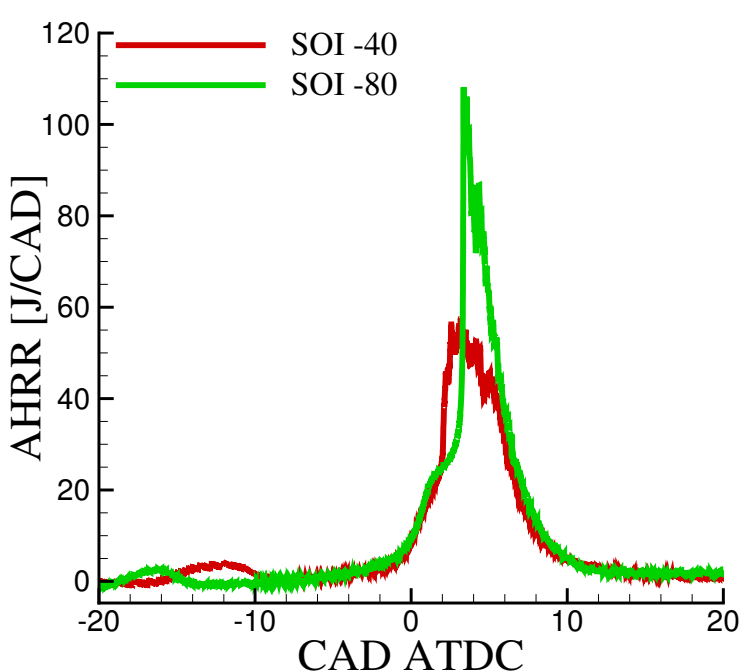

Figure 6: AHRR comparison of SOI -80 and -40 as representators of low and high mixture stratification levels, respectively.

Although it was not for the PPCI combustion, the behavior of inclination toward single stage ignition was also reported by Pei et al. [25] for highly stratified air-fuel charges, Spray A experiments [26], where almost no effects of double stage ignition and negative temperature coefficient region was observed in the numerical simulations. In PPCI combustion, observed differences of combustion phasing speeds toward second stage heat release can be considered as a key parameter to provide a better control on timing of the second stage heat release rate. Hence, the important task then would be generating a desired mixture stratification for different engine operating conditions.

Although $Z$ is a conserved scalar, its higher magnitudes represents the air-fuel mixture strength. In the stratified mixtures availability of $Z_{S t}$, due to its highest chemical reactivity, can alter the ignition and combustion phasing. In case of PPCI combustion, flame structure diagrams can provide useful insights on ignition and combustion phasing and how they are correlated to the level of mixture stratification. Simulation results of mixture fraction versus temperature for SOI of -80 and -40 ATDC were shown in Fig.7 and Fig.8, respectively.

Initially, it should be noted that there were range of mixture fractions (similar to a line) with temperature about $640 \mathrm{~K}$ for SOI -80 and $700 \mathrm{~K}$ for SOI -40 in Fig.7 and Fig.8. These mixture fractions are the fuel trapped in the piston crevice volume that will not participate in the combustion and discussions below do not take these mixture fraction into account. Moreover, temperature difference of these trapped mixture fractions in the crevice volume of the cases is due to the minor differences of the intake air temperature as stated in Table 1 for different injection timings. For crank angle -20 ATDC, it can be seen that mixture fraction (not the ones in the crevice volume) in the low stratification case of SOI -80 (Fig.7) spans a narrower range compared to the high stratification case of SOI -40 (Fig.8). This is because of a very early injection in SOI -80 , air and fuel had enough time to mix and form lean mixtures. For all shown engine times of SOI -80, mixture fractions were lower than $Z_{S t}=0.0558$.

SOI -80: At early crank angles, it can be seen that compared to SOI -40 , having more time for mixing, has caused a large amount of mixture fractions to have very similar temperature (around 750 $\mathrm{K})$. Relatively wide range of mixture fractions (from 0 to 0.035 ) simultaneously ignite and increase the temperature up to about $800 \mathrm{~K}-900 \mathrm{~K}$ as for first stage ignition around -16 CAD ATDC. Interestingly, this occurs earlier for SOI -80 compared to SOI -40 . Second stage ignition was around 5 CAD ATDC where temperature of the richer mixture fractions $(Z \approx 0.035)$ suddenly elevates to above $2000 \mathrm{~K}$. And then reaction zone also spreads for leaner mixture fractions at the later engine times. It implies that engine time needed from first to second stage ignition is 21 engine crank angles for the case of SOI -80 .

SOI -40: It can be seen both in Fig. 8 and Fig.6 that first stage ignition for SOI -40 occurs later than SOI -80 around -12 CAD ATDC. The reason for this can be attributed to the retarded injection timing of SOI -40 where cooling due to evaporation affects SOI -40 more than than SOI -80 . As mentioned, the range of ignitable mixture fractions before ignition for SOI -40 is almost double the value in SOI -80 due to lowered premixing. This has created mixtures richer than $Z_{S t}=0.0558$ which will result in higher reaction rates if the needed activation energy can be provided by compression. After the first stage ignition, availability of these richer mixture fractions with higher reactivity, as it was also mentioned in the discussion of the Fig.6, noticeably shortens the time from first stage to second stage ignition. 

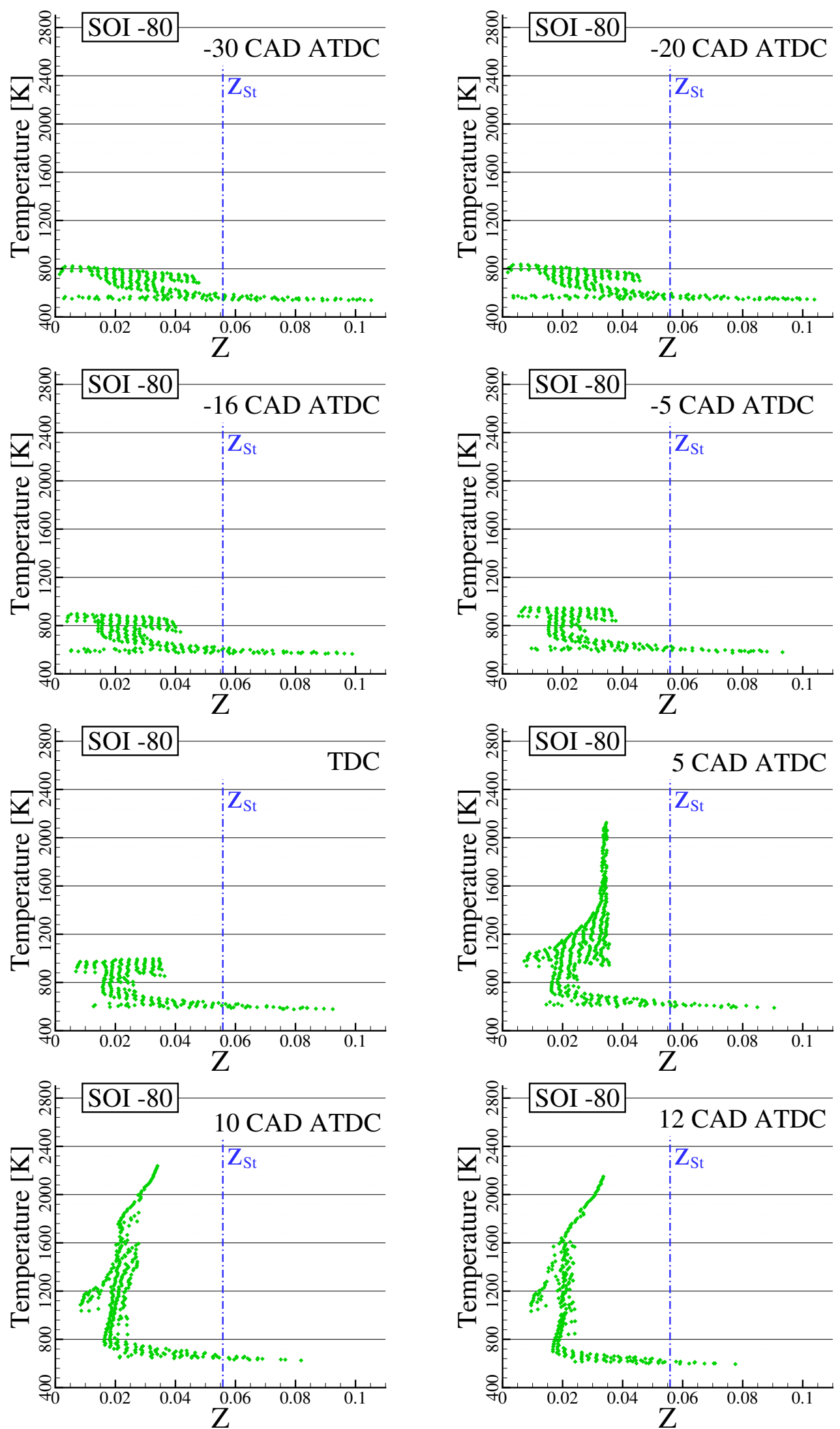

Figure 7: Z-T diagram of flame structure for the low air-fuel mixture stratification case of SOI - 80 for shown engine times. $Z_{S t}=0.0558$.

Page 12 of 16 

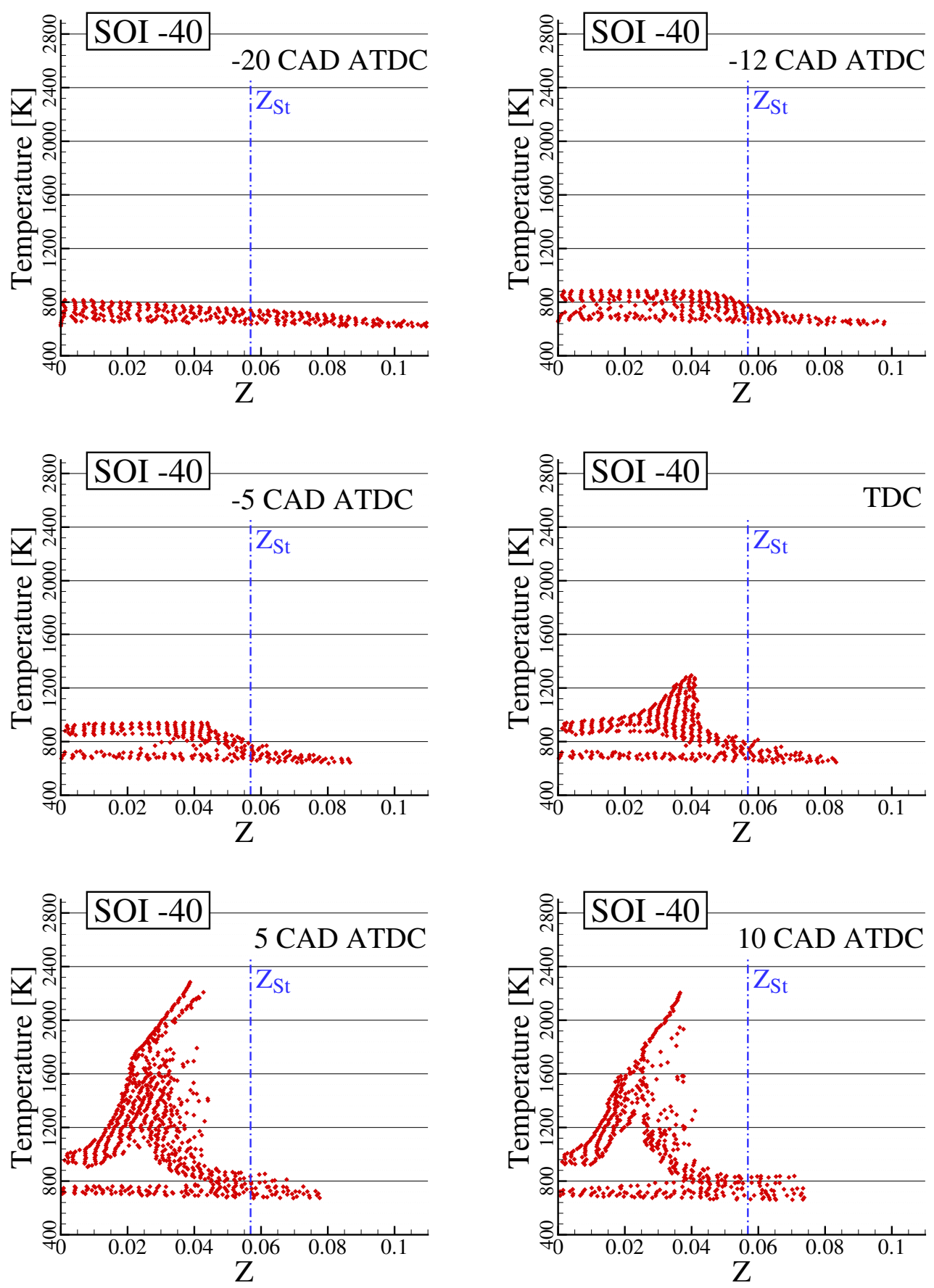

Figure 8: Z-T diagram of flame structure for the high air-fuel mixture stratification case of SOI -40 for shown engine times. $Z_{S t}=0.0558$.

Page 13 of 16 


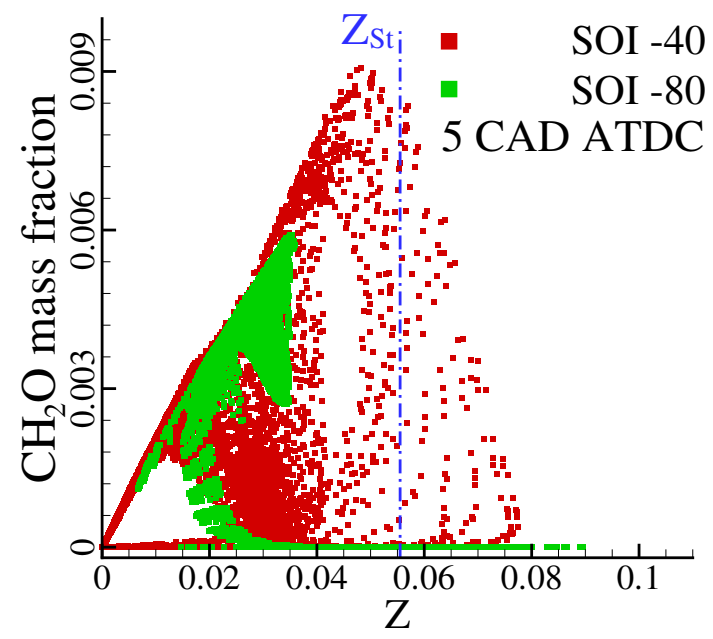

Figure 9: $\mathrm{CH}_{2} \mathrm{O}$ mass fractions of SOI -80 and SOI -40 for the whole CFD domain at 5 CAD ATDC (Second stage ignition).
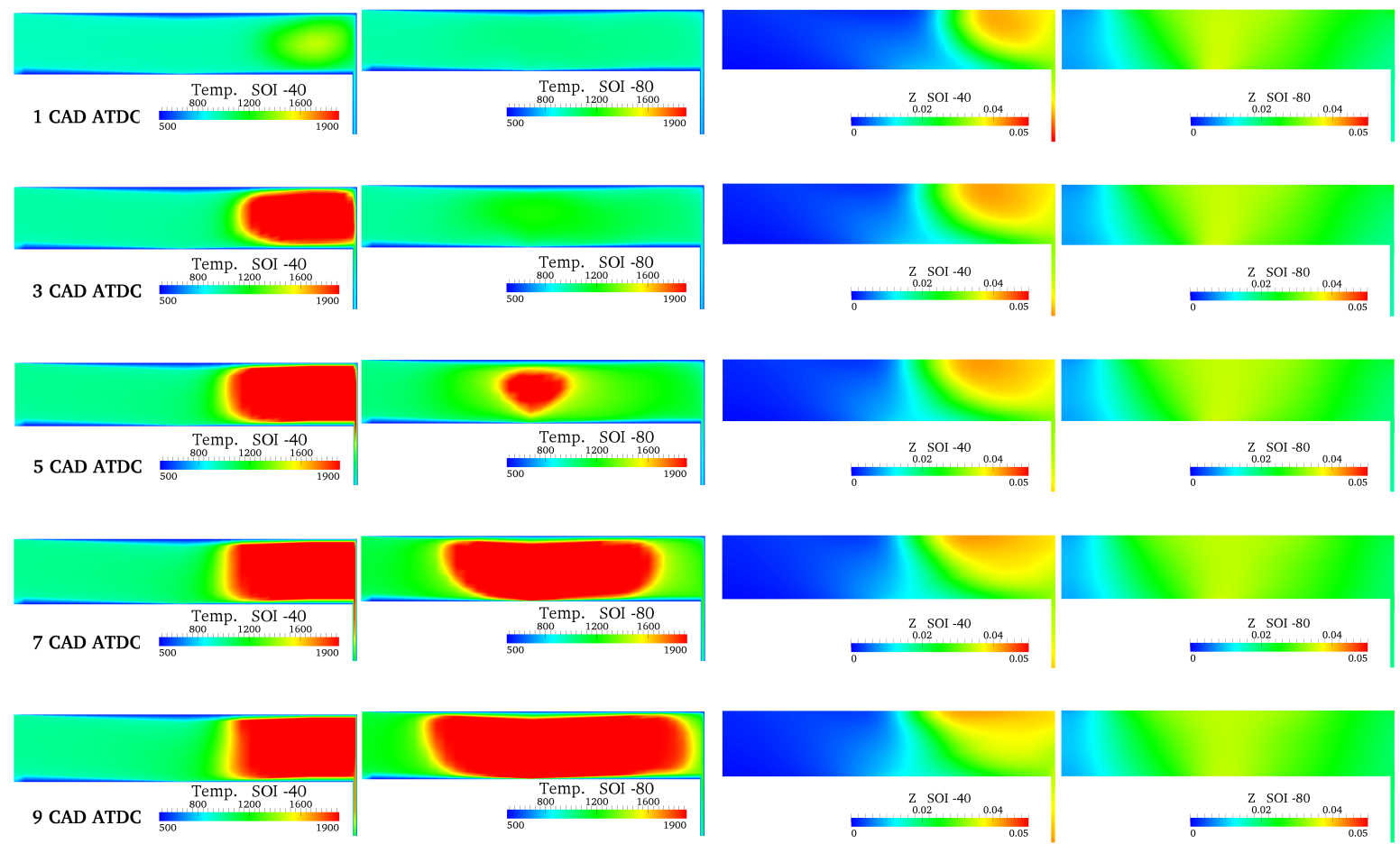

Figure 10: Temperature and mixture fraction contours of SOI -40 and SOI -80 at 1, 3, 5, 7, and 9 CAD ATDC used to discuss combustion phasing from first to second (main) ignition stages.

Page 14 of 16 
This was also shown in Fig.9 for $\mathrm{CH}_{2} \mathrm{O}$ mass fractions at 5 CAD ATDC as indicator for second stage ignition. It can be seen that due to availability of richer mixture fractions, higher $\mathrm{CH}_{2} \mathrm{O}$ was resulted for SOI -40 which shortens the transition time from first stage to second stage ignition. It represents that engine time needed from first to second stage ignition is 17 engine crank angles for the case of SOI -40. This is an important outcome of applying stratified air-fuel charges bringing in additional and effective controlling parameter which is not available in the $\mathrm{HCCI}$ engines.

On the other hand, for SOI -80 a large number of mixture fractions at $\mathrm{Z} \approx 0.03$ have higher $\mathrm{CH}_{2} \mathrm{O}$ mass fractions than SOI -40 . This is because larger amount of fuel undergo the second stage ignition in SOI - 80 which can be seen in the mixture fraction contours of Fig.10. This also causes a higher heat release rate peak in Fig.6 for SOI -80.

Explained second stage ignition characteristics of the cases can also shown in the temperature and mixture fraction contours in Fig.10. A high temperature spot was formed in SOI -40 at 1 CAD ATDC, whereas the temperature contour for the SOI - 80 is almost uniform around $1000 \mathrm{~K}$ after first ignition stage. The reason for earlier formation of this hot spot in SOI -40 can be discussed by locally richer mixture fraction of this region in Fig.10 and higher reactivity of these rich mixture fractions which increases the combustion phasing speed for SOI -40. It can also be seen from the mixture fraction contours that due to spray angle and early injection, evaporated fuel in SOI -80 had more time to mix and diffuse to the combustion chamber than SOI - 40 creating larger amount of ignitable fuel. As discussed. this was the main reason on higher AHRR peak and $\mathrm{CH}_{2} \mathrm{O}$ mass fractions for SOI -80 at second stage ignition.

\section{Conclusions}

PPCI engine experiments and simulations of the LTC concept were performed on a CI optical engine. Two cases of the low and high air-fuel mixture stratification levels were considered. Low and high air-fuel stratification levels were experimentally achieved by early (SOI -80 ATDC) and late (SOI -40 ATDC) injection timings. Experimental results show that injection around $80 \mathrm{CAD}$ has less ignition sensitivity to SOI compared to injection around -40 CAD because of lower level of stratification which was confirmed by analysis of $O H^{*}$ images. Numerical validation studies of the PPCI engine was then performed for the considered cases. Well-Mixed combustion model is used as PPCI combustion occurs in nearly homogeneous mixtures. After getting acceptable results for in-cylinder pressure, and local distribution of $O H^{*}$ within the combustion chamber of the PPCI engine, attention was given to evolution of the first and second stage ignitions and combustion phasing speed. Firstly, it was found that with the assumption of similar timing for the second stage ignition provided by intake air heating, first stage ignition occurs earlier for lower air-fuel stratification case. Secondly, high air-fuel stratification will cause in faster shift from the first stage ignition to second stage ignition of the PRF70 fuel. This was discussed to be due to higher reactivity of the mixture in high air-fuel stratification case. Combustion phasing from first to second stage ignition took 21 CAD for SOI -80 , whereas for SOI -40 this was 17 CAD. It was concluded that by generating more stratified mixtures and providing needed activation energy by engine thermal load, it would possible to have control on timing of the second stage ignition and combustion phasing speed of a PPCI engine. This study only considered low and high air-fuel stratification levels and more extensive engine experiments and simulations are needed to define a map of a PPCI engine based on injection timing (air-fuel stratification), engine IMEP or emissions, and engine operating conditions. This was considered as a future research.

\section{REFERENCES}

1. Hessel R. P. et al. "modeling iso-octane hcci using cfd with multi-zone detailed chemistry; comparison to detailed speciation data over a range of lean equivalence ratios,". SAE Technical Paper 2008-01-0047, doi: 10.4271/2008-01-0047, 2008.

2. Lechner G. A. et al. "evaluation of a narrow spray cone angle, advanced injection timing strategy to achieve partially premixed compression ignition combustion in a diesel engine,". SAE Technical Paper 2005-01-0167, doi: 10.4271/2005-010167, 2005

3. Maghbouli A. et al. "a multi-dimensional cfd-chemical kinetics approach in detection and reduction of knocking combustion in diesel-natural gas dual-fuel engines using local heat release analysis". SAE Int. J. Engines, doi: 10.4271/2013-010865:(6)777-787, 2013.

4. Epping K., Aceves S., Bechtold R., and Dec J. E. "the potential of hcci combustion for high efficiency and low emissions". SAE Technical Paper 2002-01-1923, doi:10.4271/2002-01-1923, 2002.

5. Takeda Y., Keiichi Nakagome., and Keiichi N. "emission characteristics of premixed lean diesel combustion with extremely early staged fuel injection". SAE Technical Paper 961163, doi:10.4271/961163, 1996.

6. Yanagihara H., Sato Y., and Mizuta J. "a study of di diesel combustion under uniform higher-dispersed mixture formation". JSAE review, doi:10.1016/S0389-4304(97)000313:18(4)361-367, 1997.

7. Changle L., Lianhao Y., Shamun S., Tuner M., Johansson B., Solsjo R., and Bai X. "transition from heci to ppc the sensitivity of combustion phasing to the intake temperature and the injection timing with and without egr". SAE Technical Paper 2016-01-0767, doi:10.4271/2016-01-0767, 2016.

8. Izadi M., Dam N., Somers B., and Johansso B. "ignition sensitivity study of partially premixed combustion by using shadowgraphy and oh* chemiluminescence methods". SAE Technical Paper 2016-01-0761, doi:10.4271/2016-01-0761, 2016.

9. Dempsey A. B., Curran S., Wagner R., and Cannella W. "effect of premixed fuel preparation for partially premixed combustion with a low octane gasoline on a light-duty multicylinder compression ignition engine". J. Eng. Gas Turb. Power, doi:10.1115/1.4030281:11(137)111506, 2015.

10. Tang Q., Liu H., Li M., and Yao M. "study on the double injection strategy of gasoline partially premixed combustion under a light-duty optical engine". SAE Int. J. Engines, doi: 10.4271/2016-01-2299:9(4), 2016. 
11. Tamagna D., Ra Y., and Reitz R. D. "multidimensional simulation of pcci combustion using gasoline and dual-fuel direct injection with detailed chemical kinetics". SAE Technical Paper 2007-01-0190, doi:10.4271/2007-01-0190, 2007.

12. Kokjohn S. L., Hanson R. M., Splitter D. A., and Reitz R. D. "experiments and modeling of dual-fuel heci and pcci combustion using in-cylinder fuel blending". SAE Int. J. Engines, doi: 10.4271/2009-01-2647:2(2)24-39, 2009.

13. Pal P., Keum S., and Im H. G. "assessment of flamelet versus multi-zone combustion modeling approaches for stratifiedcharge compression ignition engines". Int. J. Engine Research, doi: 10.1177/1468087415571006:17(3)280-290, 2015.

14. Kong S-C., Kim H., Reitz R. D., and Kim Y. "comparisons of diesel pcci combustion simulations using a representative interactive flamelet model and direct integration of cfd with detailed chemistry". J. Eng. Gas Turb. Power, doi: 10.1115/1.2181597:129(1)252-260, 2006.

15. Lucchini T., Della Torre A., D’Errico G., Montenegro G., Fiocco M., and Maghbouli A. "Automatic Mesh Generation for CFD Simulations of Direct-Injection Engines". SAE Technical Paper 2015-01-0376, doi:10.4271/2015-01-0376, 2015.

16. Weller H. G., Tabor G., Jasak H., and Fureby C. "A Tensorial Approach to CFD using Object Orientated Techniques". Computers in Physics, doi:10.1063/1.168744:12(6)620, 1998.

17. Lucchini T., D’Errico G., Brusiani F., and Bianchi G. "A Finite-Element Based Mesh Motion Technique for Internal Combustion Engine Simulations". COMODIA 2008, MS2-3, 2008.

18. Huh K. Y., Chang I. P., and Martin J. K. "A comparison of boundary layer treatments for heat transfer in IC engines". SAE Technical Paper 2015-01-0376, doi:10.4271/900252, 1990.

19. Maghbouli A., Lucchini T., D'Errico G., and Onorati A. "Effects of grid alignment on modeling the spray and mixing process in direct injection diesel engines under nonreacting operating conditions". Applied Thermal Engineering, doi:10.1016/j.applthermaleng.2015.07.051:91,901-912, 2015.

20. Reitz R. D. "Modeling Atomization Processes In High Pressure Vaporizing Sprays". Atomization and Spray Technology, pages 3,309-337, 1987.

21. Lucchini T., D'Errico G., and Ettorre D. "Numerical investigation of the spray-mesh-turbulence interactions for high-pressure, evaporating sprays at engine conditions". Int. J. Heat Fluid Flow, doi:10.1016/j.ijheatfluidflow.2010.07.006:32, 285-297, 2011.

22. Liu Y-D., Jia M., Xie M-Z., and B. Pang. "enhancement on a skeletal kinetic model for primary reference fuel oxidation by using a semidecoupling methodology". Energy \& Fuels, pages 26(12)7069-7083, 2012.

23. Aceves M. S. et al. "A multi-zone model for prediction of HCCI combustion and emissions". SAE Technical Paper 2000-01-0327, doi:10.4271/2000-01-0327, 2000.
24. Maghbouli A. et al. "parametric comparison of well-mixed and flamelet $n$-dodecane spray combustion with engine experiments at well controlled boundary conditions". SAE Technical Paper 2016-01-0577, doi:10.4271/2016-01-0577, 2016.

25. Pei Y. et al. "an analysis of the structure of an n-dodecane spray flame using tpdf modelling". Combustion and Flame, 168:168, 420-435, 2016.

26. Engine combustion network. http://www.sandia.gov/ ecn/index.php, 2016.

\section{Contact Information}

Amin Maghbouli

Internal Combustion Engine Group

Department of Energy, Politecnico di Milano

Via Lambruschini, 4

20156 Milano, Italy

amin.maghbouli@polimi.it

\section{Abbreviations and Definitions}

$\begin{array}{ll}\text { AHRR } & \text { Apparent Heat Release Rate } \\ \text { ATDC } & \text { After Top Dead Center } \\ \text { CAD } & \text { Crank Angle Degree } \\ \text { CI } & \text { Compression Ignition } \\ \text { EGR } & \text { Exhaust Gas Re-circulation } \\ \text { HCCI } & \text { Homogeneous Charge Compression Ignition } \\ \text { IMEP } & \text { Indicated Mean Effective Pressure } \\ \text { LTC } & \text { Low Temperature Combustion } \\ \text { MZWM } & \text { Multi-Zone Well-Mixed } \\ \text { PPCI } & \text { Partially Premixed Compression Ignition } \\ \text { SOI } & \text { Start of Injection } \\ Z & \text { Mixture fraction } \\ Z_{S t} & \text { Stoichiometric mixture fraction }\end{array}$

Page 16 of 16 